\title{
Minicomputer processing of dual Purkinje image eye-tracker data
}

\author{
JOHN E. EVANS III and JAMES C. GUTMANN \\ Department of Industrial Engineering and Operations Research \\ Virginia Polytechnic Institute and State University, Blacksburg, Virginia 24061
}

\begin{abstract}
Eye movement data analysis often entails reliable detection of the onset of saccades. Saccades are most easily separated from fixations on the basis of movement velocity and acceleration. Convolution integers are used to estimate the velocity and acceleration of eye movements, and these serve as the basis of an eye movement data-classification program. The technique of derivative estimation by convolution integers has the advantage of being easily calculated and the disadvantage of returning results shifted in phase. The disadvantage is overcome by using ring buffers for the synchronization of data and results.
\end{abstract}

Over the past several decades, there has been a steady effort to develop a convenient method of eye movement measurement. The results of this effort have yielded many different measurement techniques that vary widely in the obtrusiveness of measurement, ease of calibration, accuracy, and cost. In addition, the results of eye movement measurement may result in a film record, a videotape record, or analog voltages proportional to the $x-y$ position of the optic axis.

Once eye movement measurements have been obtained, most investigators are interested in the reliable classification of eye movement data to indicate either saccadic movement or fixation. Saccadic eye movements are characterized by high movement velocity and high initial acceleration and terminal deceleration (Ditchburn, 1973). Fixations are characterized by fairly stable eye position and low-velocity movements. The classification of eye movement data recorded on films or videotapes is often made by human observers, who view the films or videotapes of eye movement either frame by frame or at a slower speed than that used to record. The observers classify cye movements by judging the stability of eye position.

Data-reduction methods based on observer judgment are both time consuming and prone to judgment error. As a consequence several investigators (Anliker, 1976; Kundel \& Nodine, 1973; Snyder \& Taylor, Note 1) have developed computerized eye movement dataclassification techniques that take linearized eye movement data that have been digitized and sort the data according to some algorithm. One such commonly

The authors gratefully acknowledge the guidance and support of Harry L. Snyder. This research was supported by Contract F33615-76C-5022 from the Aerospace Medical Research Laboratory, Air Force Systems Command. Copies of the software mentioned in this article may be requested from the first author. used algorithm is based on the observation that fixations are characterized by relatively constant position (Lambert, Monty, \& Hall, 1974). If over some specified time interval, eye position stays within a specified error band from the mean eye position, then a classification of fixation is returned. If eye position exceeds the range of the error band, then a classification of saccade is returned. Another algorithm (Anliker, 1976) estimates velocity of eye movement and returns a classification based on a comparison of the estimated velocity with some specified velocity.

This paper includes a description of an eye movement data-classification program that estimates eye movement velocity and acceleration and uses these estimates to classify data. This program is currently being used to classify data obtained from an SRI international dual Purkinje image eye tracker that has been digitized and written out to magnetic tape.

\section{DESCRIPTION OF DATA-ACQUISITION SYSTEM}

During the course of experiments in our laboratory, subjects search for multiple alphanumeric targets displayed on a 945-line closed-circuit television monitor. During the search the $x$ and $y$ position of the subject's eye rotations are tracked by the SRI tracker. A PDP $11 / 10$ computer samples the $\mathrm{x}$ and $\mathrm{y}$ coordinates, valid position, and keypad response channels, shown in Figure 1, once every 2 msec. The sampled information is continuously written out to magnetic tape using multiple buffered asynchronous data-transfer techniques.

The information sampled from the valid position channel is used to indicate whether the $x$ and $y$ coordinate information is valid. If the eye tracker loses track lock or if the subject blinks, a $+5-\mathrm{V}$ signal is placed on the valid position channel. The keypad response 


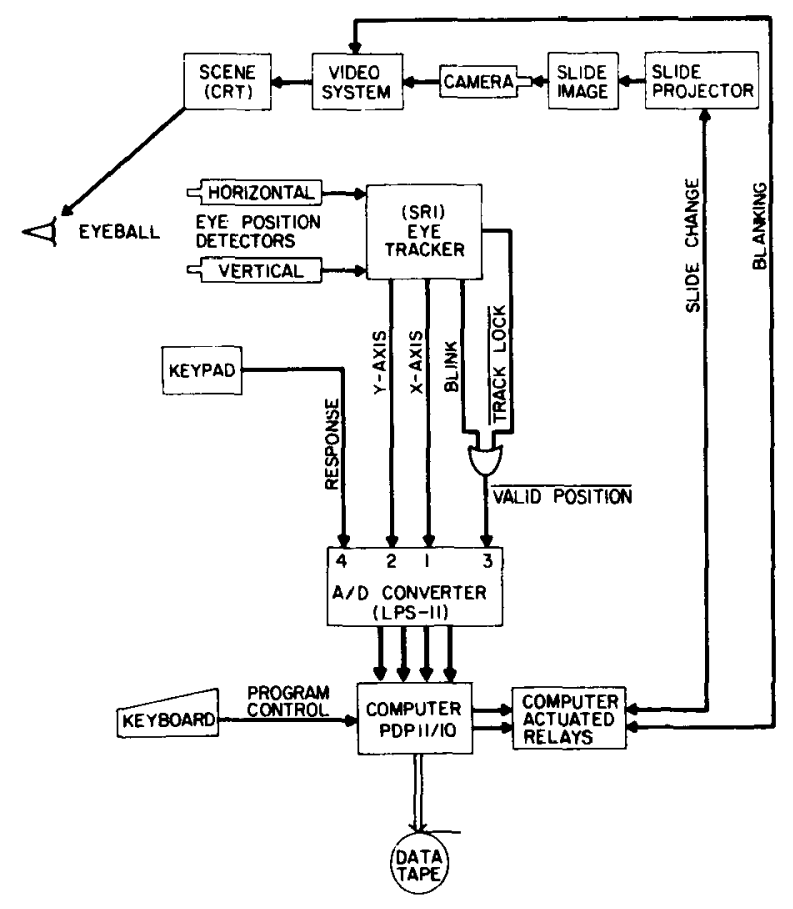

Figure 1. Block diagram of experimental data-acquisition system.

channel is used to indicate the end of the subject's search, blank the television screen, and change the target.

\section{DATA REDUCTION}

The data-reduction program used is written in FORTRAN IV and, by using a laboratory-developed tape input package for reading raw data from magnetic tape, the program may be executed on either the laboratory's PDP $11 / 10$ or PDP $11 / 55$ computers or the university's IBM $370 / 158$. The flexibility derived from machine-transportable software is particularly valuable given the resource-scheduling considerations for the laboratory computers that are utilized by several programs of research.

An overview of the data-reduction program is illustrated in Figure 2. The main program accepts and echos program-control information provided by the user and, using that information, generates a chronological eye movement classification report based on $x$ and $y$ coordinate information read from magnetic tape. Eye movement data classification is performed by subroutine CLASFY, based on estimates of the instantaneous velocity and acceleration of eye movements computed from the raw data by function CONVOL. The classification criteria are communicated through a named common block to subroutine CLASFY from user inputs accepted by subroutine SETVAL. Subroutine INRNG and function OUTRNG are used for data synchronization to facilitate the generation of the eye movement chronology report.
To utilize an eye movement classification scheme based on instantaneous velocity and acceleration of eye movements, a method for computing the first and second derivatives of the digitized eye position signals received from the eye tracker was incorporated. The derivative computation technique used convolves an integer list with a vector of raw data points. The resulting derivative estimate is the least-squares fit for the polynomial chosen (Hershey, Zakin, \& Simha, 1967; Savitsky \& Golay, 1964; Steiner, Termonia, \& Deltour, 1972). The polynomial order chosen is based on the expected form of the raw data, the selected length of the convolution integer list, and the time that can be allocated for computation. Although such lists are available for computing first and second derivatives separately, laboratory tests of known functions on which noise has been superimposed have shown that successive calculations of the first derivative have a higher noise immunity than do direct calculations of the second derivative.

By using a sliding vector of raw data points, the derivatives may be calculated for each data point rather than only at the center points of vectors that are disjoint in time. The larger number of points at which the derivatives are available makes possible data classification without a significant lag in the time between the input of raw data and the eye movement classification. The use of the convolution integers technique for calculating the derivatives of raw data generated by a function of unknown form has two advantages: (1) The order of the equation that is to be fitted to the raw data points may be selected flexibly from published lists of convolution integers without extensive programming; and (2) the computational requirements of the convolution integers technique are minimal for the accuracy provided, which makes the technique an attractive choice of method for on-line applications.

The convolution operation requires a vector of data points. Since it is desirable to produce a derivative result corresponding to each data point, a method for storing the most recently read data points for recall during the convolution operation is required. A ring buffer has the desired characteristics, and it has been incorporated within an array in function CONVOL.

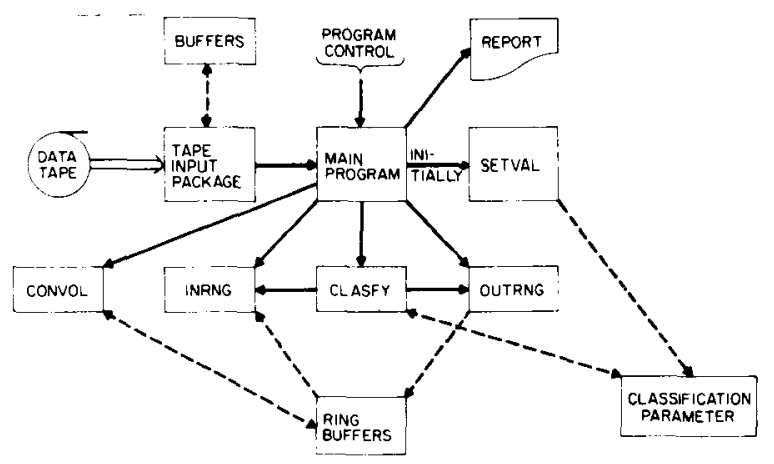

Figure 2. Flow diagram of data-analysis program. 

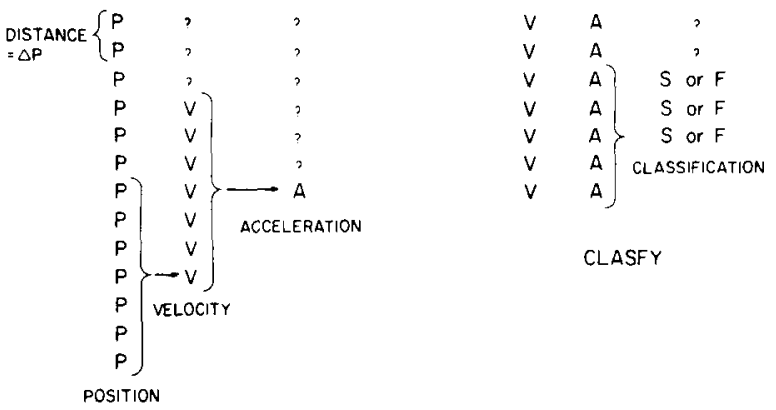

CLASFY

CONVOL

Figure 3. Synchronization of results by CONVOL and CLASFY.

The ring buffer remembers the necessary number of data points without requiring an array large enough to contain all the data points.

The convolution integers technique also has two disadvantages. First, the result returned from the convolving multiplication and normalization is associated with the center point of the vector of data points, thereby introducing a phase shift. Second, since the calculation of a result requires a "full" vector of data points, there are several data points at the beginning and end of the stream of raw data points for which there can be no associated result.

The phase shift between inputs and outputs of function CONVOL is shown in Figure 3. Before an eye movement velocity output is generated, the eye position data points must be supplied to function CONVOL until the ring buffer of Length 7 is filled. The velocity value at Subscript 4 is produced when the position value is at Subscript 7 . Similarly, the ring buffer of velocity values must fill at Subscript 10 before acceleration output is produced at Subscript 7 . The standing phase difference is therefore such that Position i results in Velocity $\mathrm{i}-1$ and Acceleration $\mathrm{i}-6$. Since a full ring buffer is required to generate an output, the initial three velocity points and the initial six acceleration points cannot be computed. Similarly, at the end of the list of eye position data points, the last three corresponding velocity points and last six corresponding acceleration points cannot be computed. The phase differences shown are only applicable for the use of a convolution integer list containing seven integers. A larger list of integers causes larger phase differences.

The first disadvantage of using convolution integers is circumvented through the use of additional ring buffers for the synchronization of data input and derivative calculation. The ring buffer (illustrated in Figure 4) is effectively an implementation of an array in which only a number of the sequentially entered recent values is available. Since function CONVOL provides calculation of the result subscript from the input subscript, the ring buffers provide a convenient method for storing several different phase-shifted results in several different ring buffers and then returning synchronized results corresponding to the smallest of the subscripts used to store the results. The second disadvantage can be negated effectively by using a sampling rate substantially higher than the anticipated frequency of the phenomenon under observation, so as to cause the significance of the "lost" data points to be negligible.

Subroutine CLASFY causes a phase differential between its instantaneous input and output similar to that of function CONVOL, as is illustrated in the right portion of Figure 3. The phase shift is caused by the use of a sliding vector of several instantaneous velocity and acceleration values. By using a vector of values rather than a single instantaneous value, a much more accurate and reliable classification as fixation or saccade is possible even in the presence of noise. Since the data vector is a sliding one, an eye movement classification is generated for each raw data point except those lost at the beginning and end of the list of eye position data points.

Subroutine CLASFY uses function INRNG to obtain sequential velocity and acceleration values that are in phase with each other. These values are separately stored in ring buffers. A sum of the values contained in each ring buffer is calculated at each time interval. If the sum of the acceleration ring buffer values and the sum of the velocity ring buffer values exceed specified threshold values obtained from subroutine SETVAL, then an eye movement classification of saccade is returned for the current time interval. If either or both of the ring buffer value sums are less than the specified threshold values, then a classification of fixation is returned instead. Thus, high-velocity and acceleration eye movements yield an eye movement classification of saccade. The inclusion of acceleration in the classification of eye movements has led to more stable and reliable results than those classifications made solely on the basis of velocity.

The report produced by the data-reduction program consists of an initial header in which the magnetic tape reel number is reported and separate partitions in which the results for each subject/experimental trial

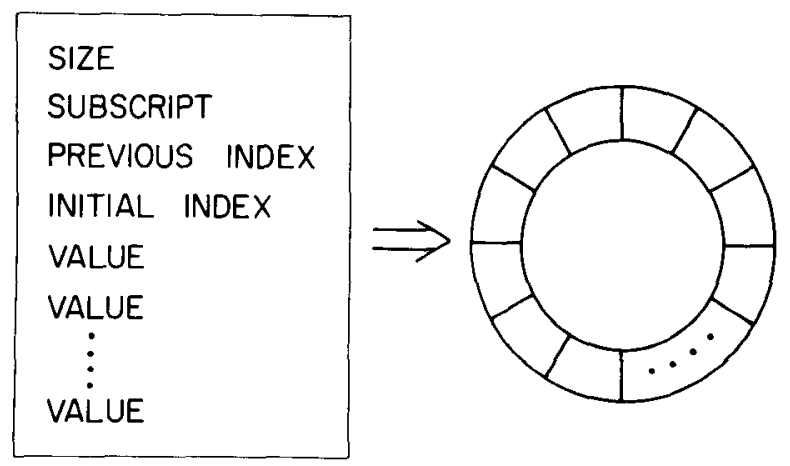

Figure 4. Diagram of ring buffer structure. 
are reported. The separate partitions each consist of a header containing the subject/trial number and labels for the output columns, the report body, which contains the chronology of eye movements, and the summary, which contains the recorded subject response and mean time and distance for fixations and saccades per experimental trial.

The chronological report of eye movements consists of a label (fixc tion, saccade, blink, ?), the initial position of the labeled event, the time elapsed during the event, the distance traveled during the event if it is a fixation or saccade and, $i_{1}$ the case of a fixation, the mean position of the fixation. The classifications are provided by subroutine CLASFY, the positions are extracted from the ring buffers by function OUTRNG, the time is calculated by counting the number of samples, and the distance is calculated as the sum of the incremental vector distances between successively sampled eye positions. The report may then be used to reconstruct accurately an idealized sequence of eye movements over any interval of time in order to provide insight into the subject's performance in the experiment.

The report also contains mean fixation and mean saccade duration and extent per experimental trial. These data are used to determine, through the use of inferential statistics, the effect of independent variables on eye movement parameters. For example, one might be interested in determining the effect of image quality on fixation duration. A group of subjects could be asked to acquire targets from several stimuli that varied in image quality. Having obtained these data, one could statistically compare mean fixation duration as a function of image quality.

\section{CONCLUSION}

The use of convolution integers for the calculation of derivatives of digitized eye position data can provide the basis of an eye movement classification scheme. The advantages of using convolution integers are that they are quickly calculated and quite accurate. When implemented as a sliding window, convolution integers may be used to provide derivative estimates for every data point with the exception of a few initial and final data points. Synchronization of data points and derivative estimates may be necessary if chronological reporting of events is desired. This synchronization may be achieved through the use of multiple asynchronous ring buffers. Although the techniques described are part of an off-line analysis program, it seems likely that on-line data analysis may also be achieved.

\section{REFERENCE NOTE}

1. Snyder, H. L., \& Taylor, D. F. Computerized analysis of eye movements during static display visual search (Report No. AMRL-TR-75-91). Wright-Patterson Air Force Base, Ohio: Aerospace Medical Research Laboratory, February 1976.

\section{REFERENCES}

ANLIKER, J. Eye movements: On-line measurement and control. In R. A. Monty \& J. E. Senders (Eds.). Eye movements and psychological processes. Hillsdale, N.J: Lawrence Erlbaum, 1976.

Ditchion,, R. W. Eye movements and visual perception. Oxford: Clarendon Press, 1973.

Hershey, H. C., Zakin, J. L., \& Simha, R. Numerical differentiation of equally spaced and not equally spaced experimental data. Industrial and Engineering Chemistry Fundamentals, 1967, 6, 413-420.

Kundel, H. L., \& Nodine, C. F. A computer system for processing eyemovement records. Behavioral Research Methods \& Instrumentation, 1973, 5, 147-152.

Lambert, R. H., Monty, R. A., \& Hall, R. J. High-speed data processing and unobtrusive monitoring of eye movements. Behavior Research Methods \& Instrumentation, 1974, 6, 525-530.

Savitsky, A., \& Golay, M. J. E. Smoothing and differentiation of data by simplified least squares procedures. Analytical Chemistry, 1964, 36, 1627-1639.

Steiner, J., Termonia, Y., \& Deltour, J. Comments on smoothing and differentiation of data by simplified least square procedure. Analytical Chemistry, 1972, 44, 1906-1909.

(Received for publication October 18, 1977; revision accepted March $30,1978$. 